\title{
A Universally Fast Adaptive Optimization of an Image Quality
}

\author{
B. Zhang \\ Department of Computer of \\ Chongqing Industry Polytechnic College, \\ Chongqing, China
}

\author{
Y. Li, W.N. Huang, X. F. Lv \\ Automatics College of \\ Chongqing Univ. of Posts and Comm. \\ Chongqing, China
}

\author{
Z.X. Xie \\ Dept. of Biomedical Engineering \\ Chingqing Medical University, \\ Chongqing, China
}

\begin{abstract}
Up to date, there are no seeing publications about fast adaptive optimization of image quality (FAO-IQ). Resolving the issue of the fast adaptive optimization of image quality needs to resolve the problem about an objective image quality assessment (IQA) and to establish a criterion on an image to be optimized. In this paper, we will propose a method to resolve these issues, i.e. 1 . introducing a universal method about an objective image quality assessment(O-IQA) to establish a criterion on an image quality optimization. 2. presenting a standardization method of an image to build a base level about FAO-IQ. 3. proposing a universally fast adaptive optimization method about an image quality.
\end{abstract}

Keywords- Universal Image Quality Assessment Funtion(UIQAF); Standardization; Adaptive; Optimization; Zadeh-X Transformation; Gray/Chroma Spectra

\section{INTRODUCTION}

Up to date, there are no seeing publications about a universally fast adaptive optimization of image quality [1]. Our published methods about image quality optimization are not an adaptive methods but a conditional scanning methods [1-2], the disturbance transformation methods [3-4] and the adaptive optimization method only suitable to scotopic vision [5]. The latter reported that the optimal transformation parameter of Zadex-X transformation is Deltaopt . 3.99*AL 0.0162 for non-standardized image. Based on the massive experimental investigations, we proposed a universal optimization transformation to fast realize the image quality optimization in this paper.

Concerning image quality assessment (IQA), a known IQA method is the structural similarity (SSIM) Index between two images [6]. This is a full reference image quality assessment (FR-IQA) method. This method has the following drawbacks: (1) It is necessary to priori assume that the reference image is a standard image. (2) It is a decimal value between 0 and 1 . That is to say, the method is only able to assess the image quality degradation and not able to assess the image quality improvement regardless of that the assessed image is an improved quality image or a degraded quality image. (3) The method can not assess a single image quality like human vision. Assessing a single image quality is referred to the problem about no reference image quality assessment (NR-IQA) [4, 6-8]. Most existing NR-IQA approaches are distortion specific and are typically limited to one or two specific types of distortions. Universal IQA (U-IQA) method that can work for various categories of distortions even improvements have hardly been explored and the algorithms available are not fully adequate in performance $[4,6-8]$. Some of existing NR-IQA approaches exactly belongs to a method with a reference image, for example, an image after the image-self to be assessed is certainly processed is considered as a reference image. We proposed a universal image quality assessment (U-IQA) function (U-IQAF) to be a tool to judge if an image quality reaches optimization in this paper.

To realize an fast adaptive optimization of image quality (FAO-IQ) is necessary to have a unified base level to be an origin (stating point). This origin just a standardize image. For 8 bit image, an image whose gray left value is equal to 0 and gray right value is equal to 255 is called a standardized image. For 24 bit color image, an image whose chromatic left value is equal to 0 and chromatic right value is equal to 255 at each color channel is called a standardized image. The Zadeh- $X$ transformation that realizes the image standardization is called an image standardization transformation (IST). The practice demonstrated that the IST has certain performance enhancing image quality.

\section{UNIVERSAL IMAGE QUALITY ASSESSMENT (U-IQA) FUNCTION (U-IQAF)}

Based on the studies for the visual parameters determining image visual quality, we constructed a universal image quality assessment function (U-IQAF) denote as $\boldsymbol{C A F}$ (comprehensive image quality assessment function) to assess an image quality as follows [1, 3-5, 9].

$$
C A F=A I E * A P C L * A H F * A B W F
$$

where AIE, APCL, $\boldsymbol{A H F}$ and $\boldsymbol{A B W F}$ are called average information entropy and average physical contrast level, average hierarchial factor and average bandwidth factor respectively(The relative calculations are ignored due to limited by the length of this article). The greater the $\boldsymbol{C A F}$ value, the better the image visual quality.

The U-IQAF method assessing image quality originates from human vision and is superior to it. This method can assess the difference among the images that can not be resolved by human vision $[3,5]$.

\section{IMAGE STANDARDIZATION}

A certain distribution of physical quantity is called a spectrum of the physical quantity, for example, energy spectrum, mass spectrum and so on. The distribution of the pixels on gray/chroma is called gray/chroma spectrum. The 
following formula is called the Zadeh-X transformation [1011].

$$
T_{\text {ist }}(i, x, y)=K \frac{O(i, x, y)-\text { Theta }}{\text { Delta }}, \quad i=1,2,3
$$

A modified Zadeh-X transformation is called IST.

$$
T_{i s t}(i, x, y)=K \frac{O(i, x, y)-\operatorname{Left}(i)}{\operatorname{Right}(i)-\operatorname{Left}(i)}, \quad i=1,2,3
$$

where Theta $\in[0,255]$ and Delta $\in[1, \infty]$ are called the transformation parameters. Right and Left are the right boundary values and the left boundary value of a gray/chroma spectrum of an image, respectively. $i=0,1,2$ represents three color components of the red channel, the green channel and the blue channel. $\boldsymbol{k}$ was called an expansion or a contraction factor, here let $\boldsymbol{k}=255$. $\boldsymbol{O}(\boldsymbol{i}, \boldsymbol{x}, \boldsymbol{y})$ and $\boldsymbol{T}_{\text {ist }}(\boldsymbol{i}, \boldsymbol{x}, \boldsymbol{y})$ represents chroma value of a pixel point $(\boldsymbol{x}, \boldsymbol{y})$ before and after image standardization transformation(IST) at $i^{\text {th }}$ channel, respectively. The image through IST possesses the full band gray/chroma spectrum. IST possesses certain image enhancement function. The practices demonstrate that an image with narrow gray/chroma spectrum can not be an image with the higher visual quality.

\section{ADAPTIVE OPTIMIZATION MODEL(AOM)}

\section{A. Experimental discoveries}

In the experimental investigation long-term, we got the following discoveries.

1. We discovered that an image with high quality has about average luminance of 127.5.

2. The result of Zadeh-X transformation with Delta $=2 * 127.5$ is the original image.

3. We also discovered that the Zadeh-X transformation can be used to improve the image quality.

4. A Zadeh-X transformation with appropriate transformation parameter, Delta, could create the optimal quality image.

5. We still discovered that Delta value used to acquire the optimal quality image depends closely on the average luminance, $\boldsymbol{A} \boldsymbol{L}$, of an image.

\section{B. adaptive optimization model(AOM)}

For standardized image, the relationship between Delta and $\boldsymbol{A} \boldsymbol{L}$, that can get an optimal quality image, should statistically defer to the following equation.

$$
\text { Delta }_{\text {pt }}=41.5838+1.67385 * A b s(\text { Theta }-A L)
$$

and

$$
\text { Theta }=\left\{\begin{array}{cc}
0, & A L \leq 127.5 \\
255, & A L>127.5
\end{array}\right.
$$

The formula (3) with (4) is called an adaptive optimization model (AOM) of an image quality for transformation parameter, Delta. This ia to say that Delta can adapt to image-self visual parameter, $\boldsymbol{A} \boldsymbol{L}$. It can computed that $=249.999875$, that is approximately equal to 255 , when $A L=127.5$.

\section{IMAGE OPTIMIZATION TRANSFORMATION (IOT)}

An image with full band spectrum is called the standardized image. The image optimization transformation (IOT) is based on image standardization transformation (IST). The following modified Zadeh-X transformation [1011] is called image optimization transformation (IOT) as follows.

$$
T(i, x, y)=k \frac{O(i, x, y)-\text { Theta }}{\text { Delta }_{\text {opt }}}+\text { Theta }, \quad i=0,1,2
$$

where

$$
\text { Theta }=\left\{\begin{array}{c}
0, A L \leq 127.5 \\
255, A L>127.5
\end{array} \quad i=0,1,2\right.
$$

and

$$
T(i, x, y)=\left\{\begin{array}{cc}
255, & T(i, x, y)>255 \\
0, & T(i, x, y)<0
\end{array} \quad i=0,1,2\right.
$$

where $\mathbf{i}=\mathbf{0 , 1 , 2}$ represents three color components of the red, the green and the blue. $\boldsymbol{k}$ was called an expansion or a contraction factor, here let $\boldsymbol{k}=255$. $\boldsymbol{O}(\boldsymbol{i}, \boldsymbol{x}, \boldsymbol{y})$ and $\boldsymbol{T}(\boldsymbol{i}, \boldsymbol{x}, \boldsymbol{y})$ represents chroma value of a pixel point $(x, y)$ before and after transformation at $\boldsymbol{i}^{\text {th }}$ channel, respectively. $\boldsymbol{A L}$ is the average luminance of an image. An image through IOT possesses the optimal image quality.

\section{RESULTS}

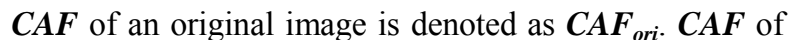
an image through optimization transformation is denoted as

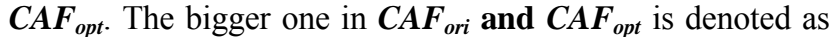

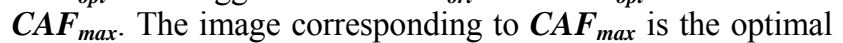
quality image.

\section{A. Image with too low luminance}

Fig. 1 a) is the image with too low luminance to be optimized, Fig. 1 b) is the standardized image of Fig 1 a). The $2^{\text {nd }}$ and $3^{\text {rd }}$ columns in table 1 are the visual parameters of the original image and the optimized image in Fig. 1,

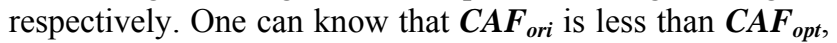
therefore $\boldsymbol{C A F}_{\text {max }}$ is $\boldsymbol{C A} \boldsymbol{F}_{\text {opt }}$. So, Fig. 1 b) is the image with optimal quality. Human vision can also resolve the quality difference between Fig. 1 a) and Fig. 1 b) like machine. 


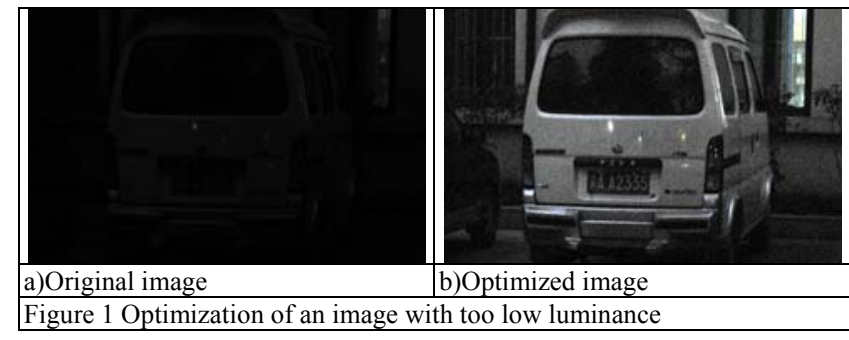

TABLE I. IMAGE QUALITY PARAMETERS

\begin{tabular}{|c|c|c|c|c|c|c|}
\hline Image & AIE & APCL & APLL & AHF & ABWF & CAF \\
\hline Fig. 1 a) & 3.7515 & $\mathbf{0 . 7 2 3 6}$ & 2.2847 & $\mathbf{0 . 2 6 3 0}$ & $\mathbf{0 . 4 6 8 8}$ & 2.7146 \\
\hline Fig. 1 b & 3.7310 & 3.9505 & 5.6614 & $\mathbf{0 . 1 0 1 6}$ & 1.0000 & 14.7393 \\
\hline Fig. 2 a) & 7.6004 & 2.3953 & 7.2290 & $\mathbf{0 . 8 5 1 6}$ & 1.0000 & 18.2052 \\
\hline Fig. 2 b) & 7.1459 & 2.4972 & 7.0751 & $\mathbf{0 . 8 5 1 6}$ & 1.0000 & 17.8447 \\
\hline Fig. 3 a) & 7.2658 & 2.0866 & 7.1312 & $\mathbf{0 . 8 6 7 2}$ & $\mathbf{0 . 8 6 7 2}$ & 15.1608 \\
\hline Fig. 3 b) & 7.0107 & 2.5817 & 7.0848 & $\mathbf{0 . 6 7 5 8}$ & $\mathbf{1 . 0 0 0 0}$ & $\mathbf{1 8 . 0 9 9 5}$ \\
\hline
\end{tabular}

\section{B. Image with normal luminance}

Fig. 2 a) is an image with normal luminance to be optimized. Fig. 2 b) is the standardized image of Fig. 1 a). The $4^{\text {th }}$ and $5^{\text {th }}$ columns in table 1 are listed the visual parameters of the original image and the optimized image of Fig.2, respectively. One can know that $\boldsymbol{C A} \boldsymbol{F}_{\text {ori }}$ is bigger than

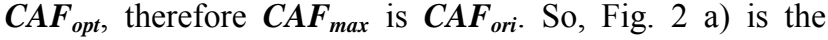
image with optimal quality. But human vision can not distinguish the quality difference between Fig. 2 a) and Fig. $2 \mathrm{~b}$ ). This situation points out that the original image is already an optimal quality image.

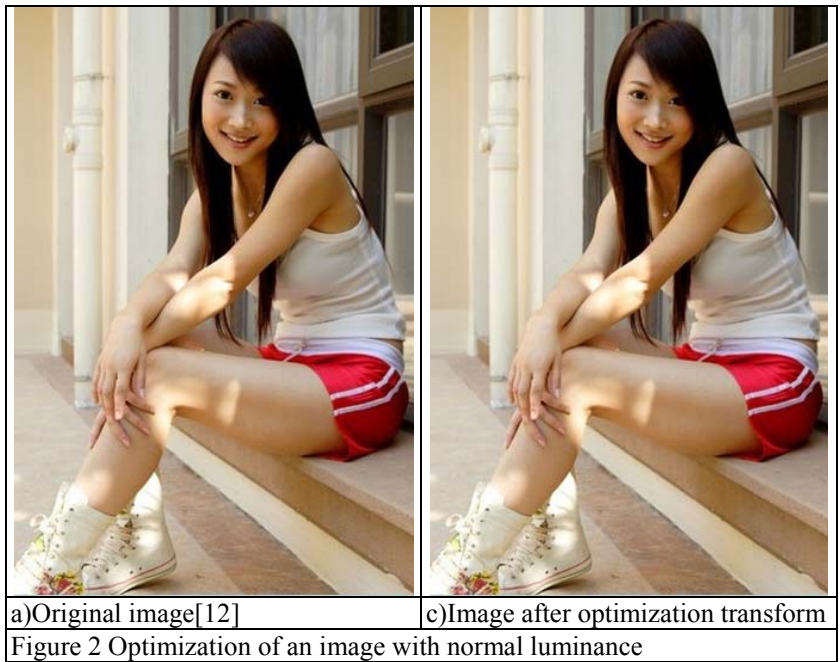

\section{Image with too high luminance}

Fig.3 a) is the image with too high luminance to be standardized. Fig.3 a) is the optimized image of Fig.3 a). The $6^{\text {th }}$ and $7^{\text {th }}$ columns in table 1 are the visual parameters of the original image and the optimized image of Fig.3, respectively. One can know that $\boldsymbol{C} \boldsymbol{A} \boldsymbol{F}_{\text {ori }}$ is less than $\boldsymbol{C} \boldsymbol{A} \boldsymbol{F}_{\text {opt }}$,

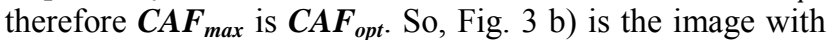
optimal quality.

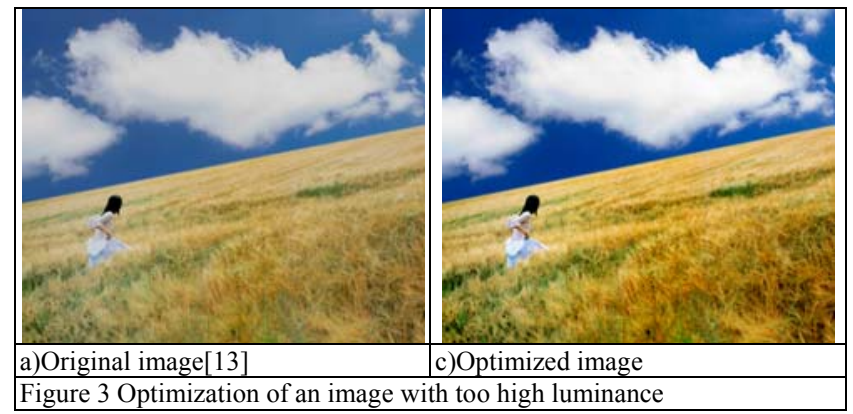

\section{CONCLUSIONS}

\section{A. Universality of the IOT}

Image optimization transformation (IOT) here proposed possesses the universality. Regardless of an image with too high luminance or an image with too low luminance even an image with normal luminance, using the method based on formulae of (5) to (7) can always get an optimal quality image, statistically, shown as Fig. 1 to Fig. 3. Universally fast adaptive optimization method here proposed is simple, speedy, so it is suitable to real time video quality optimization.

\section{B. On digital assessment method of image quality}

The digital assessment method of image quality here proposed is an objective assessment method. It origins from human vision and is superior to human vision because human vision is more rough. The image quality difference can not be revolved by human vision but by the method here proposed such as Fig. 2. So, the digital assessment method of image quality, i.e. the formula (1), can help to judge whose quality is better. The IQA method here proposed is intrinsically NR-IQA method, and can be used to assess a single image quality. The Image optimization transformation (IOT) here proposed can be considered a disturbance transformation [4]. The original image just is an optimized image if the disturbed image quality $(\mathrm{CAF}=\mathbf{1 7 . 8 4 4 7})$, which is assessed by $\boldsymbol{C A F}$, is worse than the original $(\mathrm{CAF}=\mathbf{1 8 . 2 0 5 2})$.

\section{ACKNOWLEDGMENT}

This work is supported by National Natural Science Foundation of China (Grant No.60975008), gratefully.

Corresponding author: Z. X. Xie, Email: bmezxxie@163.com

The first author: Bo Zhang, Email: cqipcmail@163.com

\section{REFERENCES}

[1] Z. X. Xie, Z. F. Wang, X. L. Xiong, Q. Hu. Color Image Quality Assessment Based on Noise Model of Human Vision Perception and Color Image Quality Optimization. Journal of Image and Graphics. 15(10): 1454-1464, 2010

[2] F. B. Tian, Z.X. Xie, X.F. Lv, J.J Cheng. Scotopic Visual Image Mining Based On NR -IQAF. 2010 International Conference on BioInspired Systems and Signal Processing (ICBSSP). Pp. 35-38, 2010.

[3] Z. X. Xie, Z.F. Wang. Color Image Quality Assessment Based on Image Quality Parameters Perceived by Human Vision System. 2010 
International Conference on Multimedia Technology, ICMT 2010. pp. $1-4,2010$.

[4] Z. X. Xie, Z. F. Wang, X.F. Lv. Single Image Quality Assessment and Creation of the Best Quality Image. 2nd International Conference on Information Science and Engineering, ICISE2010 - Proceedings. PP. 4022-4025, 2010.

[5] Z. X. Xie, Z. F. Wang, X.F. Lv. Adaptive and Automatic Acquirement of Optimal Quality Images in Lower Level Image Mining. 2010 3rd International Congress on Image and Signal Processing (CISP2010). Pp: 2322-2326, 2010.

[6] Ye Peng, D. Doermann. No-Reference Image No-Reference Image Quality Assessment Using Visual Codebooks. IEEE Transactions on Image Processing, 21( 7): 3129 - 3138, 2012.

[7] Fei Gao, Xinbo Gao, Xuelong Li, Dacheng Tao, Lihuo He, Wen Lu. Universal no reference image quality assessment metrics based on local dependency. Proceedings-2011 First Asian Conference on Pattern Recognition (ACPR), Page(s): 298 - 302, 2011.

[8] Z. Wang and A.C. Bovik, "Modern Image Quality Assessment", Morgan \& Claypool Publishers, San Rafael, CA USA, 2006.

[9] Z. X. Xie, Z.X, Q. Hu, Z. F. Wang, Y. H. Liu. A NR-IQA based on product of information entropy and contrast. Proceedings-2008 International Symposium on Information Science and Engineering (ISISE), 2008. Vol. 2, pp. 608-611

[10] Z. X. Xie, Y. Wang, Z. F. Wang, et al. "An image hiding and mining technique based on Zadeh-X tansformation," Chinese Journal of Medical Physics, vol. 24, no. 1, pp.13-15, 2007.

[11] Z. X. Xie, Y. Wang, Z. F. Wang, et al. "Hiding and mining method of lower layer image and an apparatus adopting the method," China Innovation Patent, NO: ZL 200610054379.X, 2008.

[12] This picture is taken from WEB

[13] This picture is taken from a screen protection image set. 